INVESTIGACIÓN

\title{
LOS FONDOS DE EMPLEADOS Y SU IMPORTANCIA EN LA CONSTRUCCIÓN Y RECONSTRUCCIÓN DE LA EDUCACIÓN SOLIDARIA
}

\section{THE FUNDS OF EMPLOYEES AND THEIR IMPORTANCE IN THE CONSTRUCTION AND RECONSTRUCTION OF SOLIDARY EDUCATION}

\author{
MSc.Blanca Mery Velasco Burgos ${ }^{\mathrm{a}}$, Ph.D. Maribel Cárdenas García ${ }^{\mathrm{b}}$, MSc.Jorge de Jesús \\ Cañizares Arévalo ${ }^{c}$ \\ ${ }^{a}$ Universidad Francisco de Paula Santander Ocaña, grupo de investigación Rotã \\ Carrera 29a No.10-09 Urbanización Villas de Antón Casa No.2, Ocaña, Colombia, \\ bmvelasco@ufpso.edu.co \\ ${ }^{\mathrm{b}}$ Universidad Francisco de Paula Santander Ocaña, grupo de investigación Rotã \\ Avénida 9 ${ }^{a}$ No.15-22 Barrio el Páramo, Cúcuta, Colombia, mcardenasg@ufpso.edu.co \\ ${ }^{c}$ Universidad Francisco de Paula Santander Ocaña, grupo de investigación Rotã \\ Transversal 30 No.10-50 Barrio Buenos Aires, Ocaña, Colombia, jjcanizaresa@ufpso.edu.co
}

Fecha de recepción: 02-05-2017

Fecha de aprobación: 22-06-2017

\begin{abstract}
Resumen: El artículo tiene como propósito determinar la situación actual de los fondos de empleados del municipio de Ocaña, mediante el análisis del cumplimiento del Proyecto Educativo Socio Empresarial y de la distribución de excedentes. La metodología aplicada fue descriptiva con el apoyo de la investigación documental y de campo. Como principal resultado, los fondos de empleados han colaborado en satisfacer necesidades a sus asociados, estableciéndose que la recreación con el $35,9 \%$ es la más importante, seguido por la salud con el $28,2 \%$, el $23,1 \%$ la educación y el $12,8 \%$ el desempleo. Para lo cual, se evidencia la importancia de la construcción y deconstrucción de la educación solidaria.
\end{abstract}

Palabras clave: Economía Solidaria, Educación Solidaria, Fondos de Empleados.

Abstract: The purpose of this article is to determine the current situation of the employee funds of the municipality of Ocaña, by analyzing the compliance with the Educational Business Partner Project and the distribution of surpluses. The applied methodology was descriptive with the support of the documentary and field research. As a main result, employee funds have collaborated in meeting needs of their associates, establishing that recreation with $35.9 \%$ is the 
most important, followed by health with $28.2 \%$, education $23.1 \%$ and $12.8 \%$ unemployment. For this, the importance of the construction and deconstruction of solidarity education is evidenced.

Keywords: Solidarity Economy, Solidarity Education, Employee Funds.

\section{INTRODUCCIÓN}

En Colombia existen tres tipos de organizaciones de economía solidaria: Cooperativas, Fondos de Empleados y las Asociaciones Mutuales (Organizaciones Solidarias, 2017). Es de resaltar que el presente artículo abordará los fondos de empleados en el municipio de Ocaña, específicamente su situación actual y el cumplimiento del Proyecto Educativo Socio Empresarial PESEM.

El gobierno nacional año a año ha establecido políticas públicas orientadas a la educación cooperativa y el fortalecimiento de la cultura solidaria, teniendo en cuenta aspectos como la productividad, la competitividad, la sostenibilidad y la asociatividad, como parte fundamental en el desarrollo de competencias sociales y culturales de las organizaciones que convergen el sector solidario en Colombia.

Es así como se asume la utopía de la educación y la pedagogía como una perspectiva global que permita avanzar en la construcción de competencias para la generación de conocimiento dentro de quienes hacen parte de las organizaciones de economía solidaria en este caso los fondos de empleados.

En ese mismo sentido, la pertinencia social ha sido estudiada por el profesor e investigador chileno Antonio Elizalde Hevia, citado por (Universidad Santo Tomás, 2005), a partir de un concepto de educación en y para la solidaridad:
... tiende a desbordar los límites de la educación escolarizada tradicional y es útil en múltiples ámbitos: movimientos sociales, organizaciones comunitarias, diversos colectivos, conjugando la formación científica y la educación en valores y considerando el sistema enseñanza-aprendizaje como un proceso dinámico y participativo que abarca las esferas de la salud, la economía, la política, la cultura, entre otras.

Partiendo de una educación solidaria que busca superar una concepción puramente economicista del desarrollo que contempla un mundo cada vez más interdependiente $\mathrm{y}$ garantiza un desarrollo sostenible, no solo para el presente, sino también para el futuro en toda su riqueza económica y ecológica. Una educación para la solidaridad que persigue la implicación de todos los sectores sociales en la construcción de una nueva sociedad multicultural, tolerante e igualitaria. Orientada hacia el compromiso y la acción transformadora y con un fuerte componente autocrítico hacia las propias posiciones, hábitos $\mathrm{y}$ valores. ${ }^{1}$ (p.14).

Por las consideraciones anteriores, la educación solidaria va mucho más allá

\footnotetext{
1 Tomado del libro Inventario Sistematizado de Experiencias en Educación Solidaria en Colombia. Elizalde Hevia, Antonio. Reflexiones sobre pedagogía, educación y cultura solidaria. Pp.82-100. Derecho a Solidarizarse. Memorias del Primer Congreso Internacional de Pedagogía y Cultura Solidaria.
} 
de ser solo unos cursos de cooperativismo o de inducción cooperativa, deberá generar espacios que contribuyan a la formación integral de los asociados orientados a alcanzar la eficacia y eficiencia de las organizaciones, permitiendo así cambios en la persona humana hacia la búsqueda del bienestar común, el desarrollo local y la transformación del capital humano en lo social, económico, cultural y político.

Por otra parte, en aras de logar mayor conocimiento de los fondos de empleados desde su definición, origen normativo, importancia y características contempladas en la ley 79 de 1988, ley 454 de 1998 y la actual ley 1391 de 2010 que regula los fondos de empleados y modificó el decreto reglamentario 1481 de 1989 . Es necesario realizar un recorrido normativo y teórico de los fondos de empleados con el fin de entender su accionar dentro de las organizaciones de Economía Solidaria en Colombia.

El fondo de empleados, es una entidad perteneciente al sector de la economía solidaria, de derecho común, sin ánimo de lucro, constituida por personas libres, trabajadores dependientes y subordinados de la misma empresa, que ofrecen servicios de crédito a costos mínimos. (Organizaciones Solidarias, 2017)

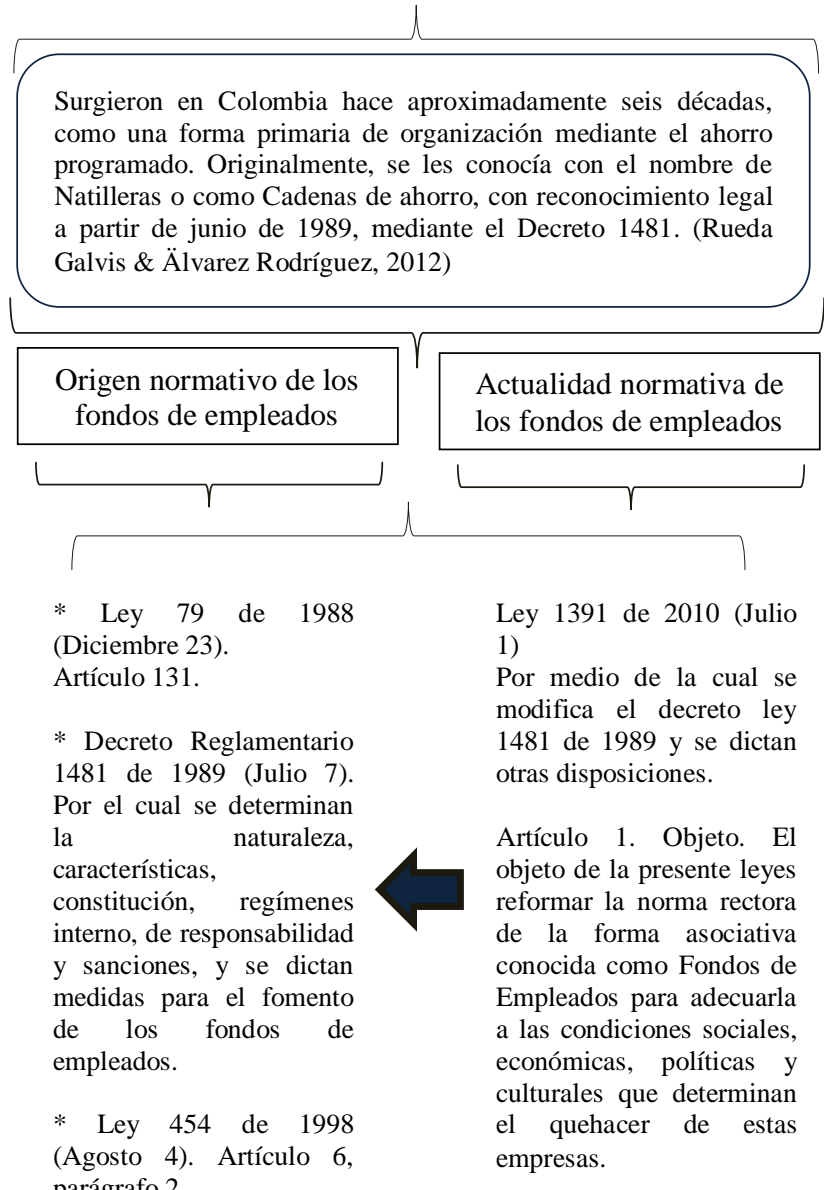
parágrafo 2 .

\section{IMPORTANCIA}

Doble naturaleza de los Fondos de Empleados El Fondo de Empleados debe caracterizarse por cumplir simultáneamente la tarea de asociar un grupo de personas que tienen necesidades y objetivos comunes y en la medida que se puedan satisfacer las necesidades, se avanza en la construcción del balance social; igualmente, deben ser muy hábiles desde el punto de vista empresarial, siendo la única manera de construir valor agregado a largo plazo. De ahí la capacidad del grupo directivo y gerencial en lograr el equilibrio entre lo social y empresarial, constituyéndose el secreto de la gestión social empresarial solidaria, la razón de existir de estas organizaciones. (FUNSSOL , 2017)

\section{CARACTERÍSTICAS}

1. Se constituyen básicamente con trabajadores asalariados.

2. Su asociación y retiro es voluntario.

3. Garantiza la igualdad de derechos de participación y decisión de los asociados sin consideración a sus aportes.

4. Presta servicios a favor de sus asociados

5. Establece la irrebatibilidad de las reservas sociales y en caso de liquidación, la del remanente patrimonial.

6. Destina sus excedentes a la prestación de servicios de carácter social.

7. El patrimonio debe ser variable e ilimitado.

8. Su constitución debe tener una duración indefinida.

9. Fomentar la solidaridad y los lazos de compañerismo entre asociados. (Organizaciones Solidarias, 2017)

Figura 1. Recorrido Normativo y Teórico de los Fondos de Empleados Fuente. Elaboración Propia a partir de los documentos citados en la figura 
Ahora bien, la educación solidaria y cooperativa se ha desarrollado en las diferentes organizaciones de economía solidaria a través de los comités de educación, quienes generan diferentes programas para la capacitación de los asociados y afiliados, sin embargo es necesario repensar sobre las metodologías y estrategias didácticas aplicadas en los procesos formativos, teniendo en cuenta que se debe llegar al equilibrio entre lo empresarial y lo social y el engranaje de la educación al proyecto empresarial de las cooperativas, para así articular el Proyecto Educativo Social Empresarial- PESEM al contexto real de la organización y que éste sea una herramienta elemental para el desarrollo integral de los fondos de empleados.

En efecto, la Ley 454 de 1998 (Colombia, Congreso de la República (1998, 4 agosto), 1998) en su artículo 5 enuncia los fines de la economía solidaria, así:

La Economía solidaria tiene como fines principales:

1. Promover el desarrollo integral del ser humano.

2. Generar prácticas que consoliden una corriente vivencial de pensamiento solidario, crítico, creativo y emprendedor como medio para alcanzar el desarrollo y la paz de los pueblos.

3. Contribuir al ejercicio y perfeccionamiento de la democracia participativa.

4. Participar en el diseño y ejecución de planes, programas y proyectos de desarrollo económico y social.

5. Garantizar a sus miembros la participación y acceso a la formación, el trabajo la propiedad, la información, la gestión y distribución equitativa de beneficios sin discriminación alguna.

Por consiguiente, se resalta el numeral 4 de lo expuesto anteriormente, en lo que corresponde a la ejecución de planes, programas y proyectos de desarrollo económico y social. Las organizaciones de economía solidaria, específicamente el objeto de estudio del presente artículo, los fondos de empleados han tenido el compromiso con sus afiliados en la consolidación de un PESEM que incorpore el plan de desarrollo de la organización junto con sus ejes estratégicos que les permita ser competitivos en el mercado, mejorar la calidad de vida, dar respuesta a las necesidades colectivas y potencializar la gestión empresarial incorporando aspectos como la educación orientada a la formación y capacitación, mediante la investigación y puesta en marcha elementos que permitan la extensión y la proyección social.

En este propósito, ( Rosales Demetrio, 2016), ha citado la concepción del PESEM desde la perspectiva de la Superindencia de la Economía Solidaria,

Según la Directiva 031 de 2000. Toda organización solidaria debe contar con un plan de desarrollo que atienda sus necesidades reales y que potencialice la gestión de la empresa asociativa en procura de la buena marcha de la misma y del mejoramiento de la calidad de vida de los asociados y de su entorno. Este plan estará sustentado en un Proyecto Educativo SocioEmpresarial, el cual consiste en el proceso permanente que orienta la ejecución del plan de desarrollo a través de actividades educativas enmarcadas en los ámbitos de 
investigación, promoción, formación, capacitación y asistencia técnica... La organización solidaria llevará a cabo un balance social que permita establecer el impacto de la ejecución del plan de desarrollo, el cual será enviado a la entidad competente cuando esta lo requiera. El balance social incluirá indicadores específicos de evaluación de la gestión educativa.

El Proyecto Educativo Social y Empresarial -PESEM- debe darse a nivel de cada empresa asociativa o de manera integrada entre varias organizaciones. (p.62).

Lo anterior permite fundamentar el estudio de la importancia del PESEM en las organizaciones de economía solidaria y para ello es necesario conocer su funcionabilidad y operatividad dentro de la organización, teniendo en cuenta que es su carta de navegación que le permitirá proyectarse en un período de tiempo y evaluar metas logradas y proyectos ejecutados. En consecuencia, se genera un interrogante por conocer el estado actual del PESEM en los fondos de empleados del municipio de Ocaña?, teniendo en cuenta que se encuentran registrados en la Superintendencia de la Economía Solidaria seis (Tabla 1).

Por lo tanto, los propósitos de la investigación se desarrollaron en el diagnóstico de la situación actual de los fondos de empleados del municipio de Ocaña, en el análisis en el cumplimiento del Proyecto Educativo Socio Empresarial y de la distribución de excedentes por parte de los fondos de empleados del municipio de Ocaña.

En tal sentido, la (Universidad Santo Tomás, 2005), cita al filósofo John Dewey “... La educación consiste en construcción y reconstrucción de experiencias". (p.31).

Tabla 1. Número de Fondos de Empleados del Municipio de Ocaña

\begin{tabular}{llc}
\hline \multicolumn{1}{c}{ FONDO DE EMPLEADOS } & \multicolumn{1}{c}{ SIGLA } & ASOCIADOS \\
\hline $\begin{array}{l}\text { FONDO DE AHORRO Y CREDITO EMPLEADOS COLEGIO } \\
\text { FATIMA }\end{array}$ & FACECOLFA & 102 \\
FONDO DE AHORRO Y CREDITO DE EMPLEADOS DE LA & FAYCEN \\
NORMAL NAL FCO FDEZ DE CONTRERAS & & 150 \\
FONDO DE EMPLEADOS DE LA RAMA JUDICIAL Y DEL & FEMPOJUDICIAL & 23 \\
MINISTERIO PUBLICO DE OCANA & & 56 \\
FONDO DE EMPLEADOS Y PENSIONADOS DE CENTRALES & FEPECENS & 81 \\
ELECTRICAS DEL NORTE DE SANTANDER & & 101 \\
FONDO DE EMPLEADOS DEL INSTITUTO TECNICO & FEITI \\
INDUSTRIAL & FACEPRUO \\
\hline FONDO DE EMPLEADOS DE LA UFPSO & & \\
\hline
\end{tabular}

Fuente. Elaboración propia a partir de datos tomados de (Super Intendencia de Economía Solidaria, 2016)

\section{METODOLOGÍA}

El nivel que se llevó a cabo para el desarrollo de la investigación fue descriptivo, con el cual se pretendió analizar la situación actual, el cumplimiento del
Proyecto Educativo Socio Empresarial y de la distribución de excedentes de acuerdo a las disposiciones normativas de la economía solidaria. 
El diseño de la investigación es documental y de campo, para lo cual, la información se recolectó mediante trabajo de campo con el instrumento de encuestas aplicado a 39 asociados de los seis (06) fondos de empleados existentes en el municipio de Ocaña según datos de la Superintendencia de Economía Solidaria.

A los efectos de éste las técnicas e instrumentos de recolección de datos aplicados en la investigación, se desarrollaron conforme a los diseños de investigación tanto documental como de campo, para lo cual, la técnica de la investigación documental fue el análisis documental de la información de los fondos de empleados como objeto de estudio y el instrumento utilizado para dicho análisis se soportó en la ficha bibliográfica, para el caso del desarrollo de la investigación de campo, las técnicas utilizadas fueron la observación: no estructurada y la encuesta: escrita y como instrumentos el diario de campo y el cuestionario. (Arias, 2004, pág. 66).

\section{RESULTADOS}

\section{DIAGNÓSTICO DE LA SITUACIÓN ACTUAL DE LOS FONDOS DE EMPLEADOS DEL MUNICIPIO DE OCAÑ}

Una vez aplicados los instrumentos a los seis fondos del municipio de Ocaña con una muestra de 39 asociados, se tabularon y se procedió al análisis de los resultados, expresados a continuación. Es de resaltar que para el caso específico del diagnóstico de la situación actual al finalizar el presente aparte se presentará la matriz DOFA a manera de colofón.

El $97,4 \%$ de los asociados a los fondos del municipio de Ocaña, participa activamente en las elecciones de la Junta
Directiva y el Comité de Control Social. Solo un asociado manifestó no participar.

El $97,4 \%$ de los asociados, reconocen que la filosofía del cooperativismo es motivación para pertenecer a los fondos de empleados $\mathrm{y}$ consideran que quienes se inscriben en las planchas para ser elegidos, conocen con claridad las responsabilidades de cada cargo.

El $84,6 \%$ de los asociados, consideran que los fondos cuentan con buenas estrategias para captar más asociados, mientras que el $15,4 \%$ considera que no. El $94,9 \%$ de los asociados, manifiestan que los servicios de los fondos de empleados son los adecuados y manifiestan tener sentido de pertenencia por el fondo. Solo 2 personas manifiestan no tenerlo. El $92,3 \%$ de los asociados, manifiestan que el control ejercido por el Comité de Control Social es el más adecuado. Solo 7,7\% manifiestan que no.

El $100 \%$ de los asociados a los fondos de empleados, creen en el control ejercido por la revisoría fiscal. El $71,8 \%$ de los asociados, consideran que los fondos buscan estrategias con otras entidades para generar recursos. De igual manera, los asociados conocen que el fondo cuenta con el apoyo de otras empresas para el desarrollo de sus actividades, por lo tanto, los asociados consideran que las alianzas estratégicas son importantes a la hora de fortalecer el desarrollo de las actividades de los fondos de empleado y las fuentes de financiación y apoyo financiero de los fondos.

Por otra parte, se establece con un $79,5 \%$, que los fondos de empleados cuentan con una buena oferta de proveedores de bienes y servicios para el desarrollo de sus actividades. Solo el $20,5 \%$ considera que no existe la oferta. 
El 74,4\% de los asociados manifiestan que los fondos de empleados utilizan nuevas tecnologías como medios de comunicación como lo es, el Correo Electrónico. El 79,5\% de los asociados, conocen que la supervisión de la Supersolidaria es preventiva, solo el $20,5 \%$ determinan que no conocen dicha supervisión.

Finalmente, el 69,2\% de los asociados, manifiestan que los fondos de empleados cuentan con acompañamiento frecuente por parte de las entidades a nivel regional y nacional, para el desarrollo de las actividades de los fondos de empleados. El $30,8 \%$ manifiesta no contar con dicho apoyo.

\section{ANÁLISIS EN EL CUMPLIMIENTO DEL PROYECTO EDUCATIVO SOCIO EMPRESARIAL Y DE LA DISTRIBUCIÓN DE EXCEDENTES POR PARTE DE LOS FONDOS DE EMPLEADOS DEL MUNICIPIO DE OCAÑA.}

Para este propósito se indagó a los 39 asociados de los diferentes fondos, formulando preguntas por cada principio cooperativo obteniéndose como resultado lo siguiente:

Principio I. Membresía abierta y voluntaria. Las Cooperativas son organizaciones Voluntaria Abierta para todas aquellas personas dispuestas a utilizar sus servicios y dispuestas a utilizar sus responsabilidades que conlleve la Membresía sin discriminación de género (sexo), raza, clase social, posición política o religiosa (Cooperativa Uniminuto, s/f)

En primera medida los asociados manifestaron que su vinculación a los fondos de empleados fue de forma voluntaria en un $100 \%$. Así mismo, un $97,4 \%$ de la población y muestra, manifestaron que al momento de la vinculación a los fondos de empleados, no se exige como requisito conocimientos sobre cooperativismo, lo que conlleva a que las vinculaciones sean más significativas y se hagan de manera voluntaria.

\section{Principio II. Control democrático de}

los asociados. Las Cooperativas son organizaciones democráticas controladas por sus miembros, quienes participan activamente en la definición de las políticas y en la toma de decisiones. (Cooperativa Uniminuto, s/f)

Las encuestas dieron como resultado que posterior al proceso de vinculación, el $48.7 \% \%$ de las personas encuestadas reciben inducción sobre cooperativismo al momento del ingreso al fondo de empleado, y el $51 \%$ no la reciben.

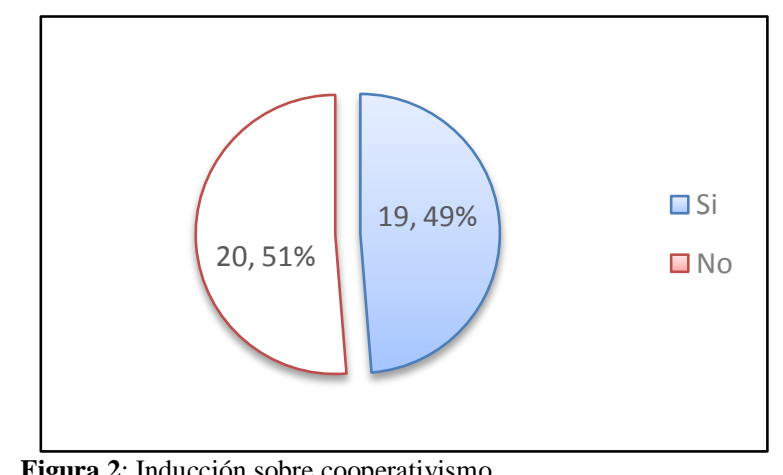

Figura 2: Inducción sobre cooperativismo

Fuente: Resultados de la Encuesta

Se identifica que el $76.9 \%$ de los asociados manifestaron que existe igual participación para hombres y mujeres en junta directiva, sin embargo, el $23.1 \%$ manifiesta que no existe igualdad de género para ocupar cargos directivos en la junta. El $76.9 \%$ de los afiliados a los fondos de empleados, conocen sus derechos como asociados al fondo, el $23.1 \%$ de los afiliados, no los conocen. 
El $74.4 \%$ de la población encuestada, manifestó que las decisiones tomadas por los miembros de las juntas directivas, son respetadas, el $25.6 \%$ manifestaron que no son respetadas. De igual manera, el $87.2 \%$ de la población encuestada, manifestaron que las decisiones tomadas por los miembros de la Asamblea General, son respetadas, el $12.8 \%$ manifestaron que no son respetadas.

Principio III. Participación económica de los asociados. Los miembros contribuyen de manera equitativa y controlan de manera Democrática el capital de la Cooperativa. Por lo menos una parte de ese capital es propiedad común de la Cooperativa. Usualmente reciben una compensación limitada, si es que la hay, sobre el capital suscrito como condición de Membresía. . (Cooperativa Uniminuto, s/f)

En relación a los créditos otorgados por los fondos de empleados, el $61.5 \%$ de los asociados consideraron que la tasa de interés son medio bajas y el $25.6 \%$ son bajas. En consecuencia se determina que los fondos de empleados manejan tasas de intereses por debajo de las entidades financieras.

El $89,7 \%$ de los asociados a los fondos de empleados, se encuentran conformes con los excedentes recibidos por ellos y que son otorgados por parte de los fondos de empleados, como resultado de las operaciones. De igual manera, el $87.2 \%$ de los asociados, consideraron que los fondos otorgan beneficios que otras entidades no suministran. Solo el $12.8 \%$ no lo consideran.

\footnotetext{
Principio IV. Autonomía e independencia. Las cooperativas son organizaciones autónomas de Ayuda Mutua, controladas por sus miembros. Si entran en acuerdo con otras organizaciones (Incluyendo el Gobierno) o tiene capital de
}

fuentes externas, lo realizan en términos que aseguren el control Democrático por parte de sus miembros y mantengan la autonomía de la Cooperativa. (Cooperativa Uniminuto, $\mathrm{s} / \mathrm{f})$

El $97.4 \%$ de los asociados, conocen que los fondos de empleados tienen convenios con otras entidades, por lo que se constituye que existe una buena gestión por parte de estos. Por lo tanto, el $62.1 \%$ de los asociados utilizan mensualmente los servicios pactados por los convenios del fondo con otras entidades, el $24.1 \%$ los utilizan semestralmente y el $13.8 \%$ los utilizan anualmente.

El $79.5 \%$ de los asociados a los fondos, consideran que las decisiones de estos son autónomas e independientes a las de las empresas, por lo que se constituye que no existe dependencia e influencia de las empresas sobre los fondos.

\section{Principio $\quad V . \quad$ Educación,} capacitación e información. Las Cooperativas brindan educación y entrenamiento a sus miembros, a sus dirigentes electos, Gerentes y empleados, de tal forma que contribuyan eficazmente al desarrollo de sus Cooperativas. (Cooperativa Uniminuto, s/f)

El $61,5 \%$ de los asociados a los fondos, reconocen que estos imparten programas y actividades educativas semestralmente, el $12.8 \%$ anual y el $25 \%$ que nunca se hacen. El $74.4 \%$ de los asociados a los fondos consideraron que las actividades de educación son buenas y el $25.6 \%$ regulares, por lo que se requiere ampliar la frecuencia de actividades así como también más actividades a impartir. Por lo tanto, el $71.8 \%$ de los asociados a los fondos, manifestaron que las actividades educativas a los fondos son suficientes, 
aunque el $28.2 \%$ consideraron que son insuficientes.

Principio VI. Cooperación entre cooperativas. Las Cooperativas sirven a sus miembros más eficazmente y fortalecen el movimiento Cooperativo. Trabajando de manera conjunta por medio de las estructuras Locales, Nacionales, Regionales e Internacionales. (Cooperativa Uniminuto, s/f)

El $69.2 \%$ de los asociados a los fondos de empleados, no conocen si el fondo tiene afiliaciones a otras cooperativas. Sin embargo, el $97,4 \%$ de los asociados si conocen si los fondos tienen convenios con otras entidades.

Principio VII. Compromiso con la comunidad. La Cooperativa trabaja para el desarrollo sostenible de su comunidad por medio de políticas aceptadas por sus miembros.

EL $82,1 \%$ de los asociados, consideraron que la comunidad está identificada con los fondos de asociados, el $17,9 \%$ considera que no. Así mismo, el $56,4 \%$ de los asociados a los fondos de empleados, expresaron que estos si desarrollan actividades socio-culturales que impacten las comunidades de su zona de influencia, el 43,6\% consideraron que los fondos de empleados no desarrollan actividades con la comunidad.

El $89,7 \%$ de los asociados manifestaron que los fondos de empleados no realizan acciones que contribuyan a la preservación del medio ambiente, por lo que se establece que los fondos no tienen un compromiso pleno ambiental.

Los fondos de empleados han colaborado en satisfacer necesidades a sus asociados, estableciéndose que la recreación con el $35,9 \%$ es la más importante, seguido por la salud con el $28,2 \%$, el $23,1 \%$ la educación y el $12,8 \%$ el desempleo. Para lo cual, se establece que los principales objetivos de los fondos de empleados, es el aseguramiento de la recreación y la salud de sus asociados.

\section{ANÁLISIS INTERNO Y EXTERNO}

La matriz DOFA (figura 3) se constituye a partir de una selección de los factores claves de éxito dados en las debilidades, oportunidades, fortalezas y amenazas evaluadas como de impacto alto en la capacidad competitiva de la organización (Bernal Torres \& Sierra Arango, 2013, pág. 120)

\section{CONCLUSIONES.}

Es necesario ampliar la gama de servicios que ofrecen los fondos de empleados, con el propósito de incentivar la captación de nuevos asociados.

De igual manera, se requiere establecer programas de capacitación de bienestar social y desarrollo del recurso humano de las empresas que fomente la responsabilidad social.

Así mismo, es importante que los fondos de empleados mejoren los canales de información y comunicación, para ello, se debe estructurar el manejo de información para procesos de control y mejoramiento continuo.

Es pertinente ofrecer mejores tasas de interés que las demás entidades financieras para lograr mayores asociados y minimizar la dependencia de las empresas patronales 
Igualmente, se deben establecer factores diferenciadores para que la comunidad reconozca a los fondos de empleados en su zona de influencia, para lo cual se propone diseñar estrategias de mercadotecnia para lograr dicho reconocimiento.
Finalmente, es vital e importante diseñar un PESEM que fomente la independencia patronal mediante la generación de estrategias educativas para captar nuevos recursos, para ello, se propone la creación de escuelas de formación empresarial. 


\section{FINANCIACIÓN}

\section{"Impacto de las Políticas Regulatorias Contables de las Empresas del Sector}

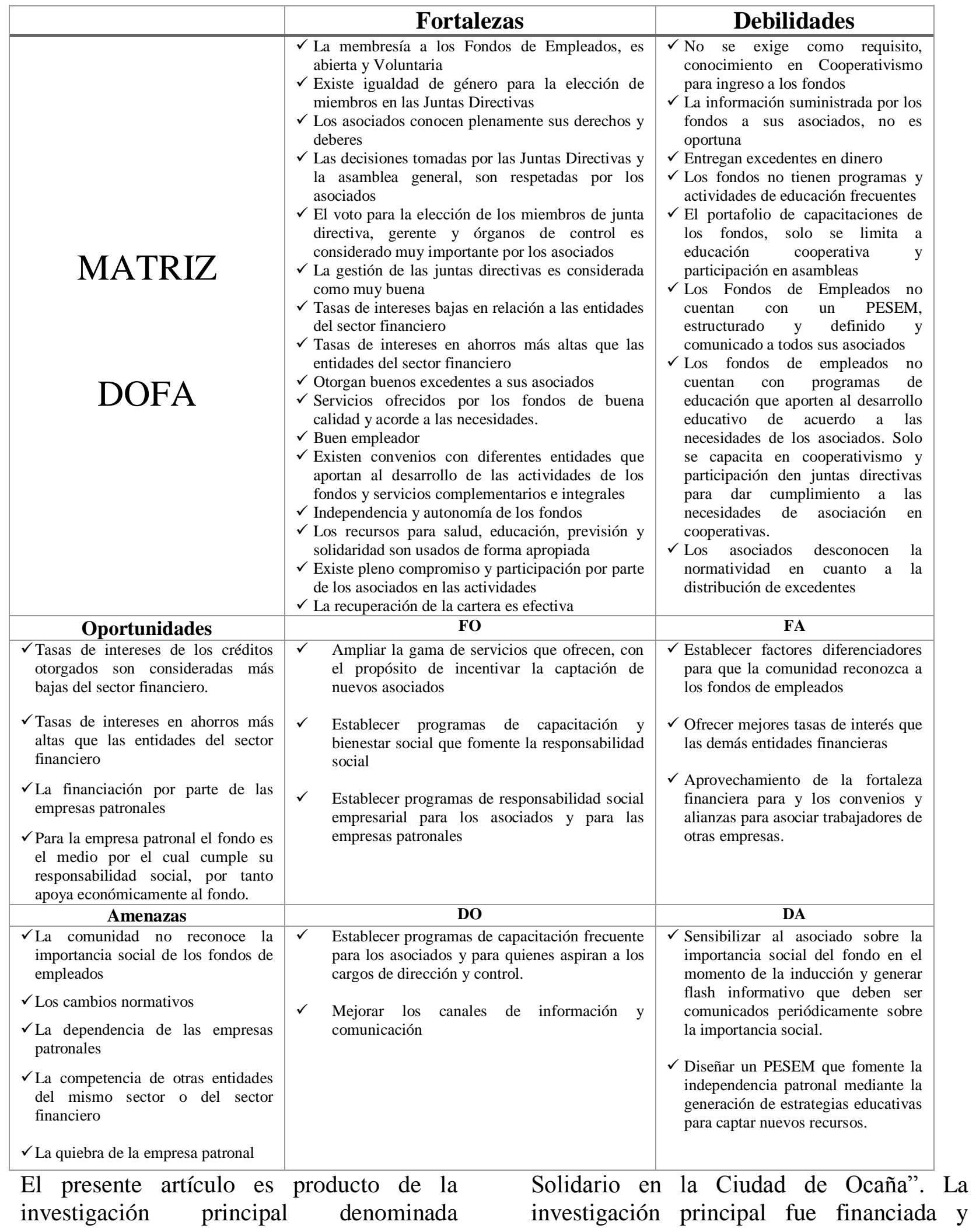


avalada por la División de Investigación y Extensión DIE de la Universidad Francisco de Paula Santander Ocaña.

\section{BIBLIOGRAFÍA}

Rosales Demetrio, C. S. (06 de 2016). Pesems estratégico en complejidad para resolver problemática tradicional de empresas de economía solidaria. Obtenido de http://www.unilibrebaq.edu.co/ojsinv estigacion/index.php/dictamenlibre/a rticle/view/713/645

Arias, F. G. (2004). El proyecto de investigación. Introducción a la metodología científica 4 Edición. Caracas: Editorial Episteme.

Bernal Torres, C., \& Sierra Arango, H. (2013). Proceso Adminsitrativo para las organziaciones del siglo XXI. Bogotá: Pearson.

Colombia, Congreso de la República (1998, 4 agosto). (04 de 08 de 1998). Ley 454 del 04 de Agosto de 1998. Por la cual se determina el marco conceptual que regula la economía solidaria, se transforma el Departamento Administrativo Nacional de Cooperativas en el Departamento Administrativo Nacional de la Economía Solidaria, se crea la Superintendencia de la Eco. Bogotá, Colombia: Diario Oficial Núm. 43357.

Cooperativa Uniminuto. (s/f). coopuniminuto.com. Recuperado el 01 de 11 de 2016, de https://www.coopuniminuto.com/ind ex.php/la-cooperativa-y-losprincipios-cooperativos

FUNSSOL . (25 de 08 de 2017). http://www.grupofep.com/basico/pdf/ fondos.pdf. Obtenido de http://www.grupofep.com/basico/pdf /fondos.pdf
Organizaciones Solidarias. (27 de 08 de 2017). Organizaciones Solidarias. Obtenido de http://www.orgsolidarias.gov.co/edu caci\%C3\%B3n-solidaria/nuestrasorganizaciones/organizacioneseconom\%C3\%ADa-solidaria/fondode-empleados

Organizaciones Solidarias. (25 de 08 de 2017). ORGANIZACIONES SOLIDARIAS. Obtenido de http://www.orgsolidarias.gov.co/edu caci\%C3\%B3n-solidaria/nuestrasorganizaciones/organizacioneseconom\%C3\%ADa-solidaria

Rueda Galvis, M. A., \& Älvarez Rodríguez, J. F. (2012). Una mirada a los fondos de empleados en Colombia. Gestión \& Sociedad, 5(2), 75-86. Recuperado el 25 de 08 de 2017, de https://revistas.lasalle.edu.co/index.p hp/gs/issue/view/52/showToc

Superintendencia de Economía Solidaria. (23 de 06 de 2016). Estadísticas. Obtenido de http://www.supersolidaria.gov.co/es/ entidad/estadisticas

Universidad Santo Tomás. (2005). Inventario Sistematizado de Experiencias en Educación Solidaria en Colombia. Bogotá: Imprenta Nacional de Colombia. 